\title{
Metodologia de Avaliação de Custos nas Universidades Públicas: Economias de Escala e de Escopo*
}

\author{
Helio Nogueira da Cruz ${ }^{* *}$ \\ Maria Dolores Montoya Diaz ${ }^{* * *}$ \\ Carlos Antonio Luque ${ }^{* * * *}$
}

Sumário: 1. Introdução; 2. Metodologia e variáveis utilizadas; 3. Resultados; 4. Considerações finais.

Palavras-chave: ensino superior; economias de escala; economias de escopo.

Códigos JEL: C23; I22.

Este artigo avaliou a existência de economias de escala e de escopo na Universidade de São Paulo, por meio da estimação da função custo multi-produto - ensino de graduação, pós-graduação, títulos de pós-graduação outorgados e publicações - a partir das informações referentes ao período 1996 a 1999 de 35 unidades de ensino e pesquisa da Universidade. Estimou-se, deste modo, um modelo para dados em painel. Os resultados indicaram claramente a existência de economias de escala produto-específicas apenas para os produtos ensino de graduação e de pós-graduação. A produção de teses e dissertações bem como de publicações apresentou retornos constantes de escala. Em termos da existência de economias de escopo, é interessante notar que no caso da USP, foram identificadas em todos produtos analisados, indicando que o modelo de universidade de pesquisa, em que se produzem conjuntamente ensino e pesquisa, também é o mais eficiente em termos de custos. Finalmente, verificou-se que o custo marginal de produção da atividade de ensino de graduação é $\mathrm{R} \$ 14,23$, do ensino de pós-graduação é de $\mathrm{R} \$ 64,79$, da produção de dissertações e teses é de aproximadamente $\mathrm{R} \$ 24.206,96$ e o da produção de trabalhos publicados em periódicos e livros nacionais e internacionais é de $\mathrm{R} \$ 3.976,02$.

\footnotetext{
*Artigo recebido em set. 2002 e aprovado em abr. 2003. Os autores agradecem as sugestões de dois pareceristas anônimos, ressaltando, porém, que os erros e omissões eventualmente remanescentes são de nossa exclusiva responsabilidade.

${ }^{* *}$ Vice-Reitor da Universidade de São Paulo e Professor Titular da Faculdade de Economia, Administração e Contabilidade/USP

${ }^{* * *}$ Prof. $^{a}$ Associada da Faculdade de Economia, Administração e Contabilidade de Ribeirão Preto/USP. E-mail: madmdiaz@usp.br

${ }^{* * * *}$ Secretário Adjunto da Secretaria de Economia e Planejamento do Estado de São Paulo e Professor Titular da Faculdade de Economia, Administração e Contabilidade/USP.
} 
A four-output cost function is used to investigate the existence of economies of scale and scope in the University of São Paulo. The following outputs were incorporated: undergraduate teaching, graduate teaching, thesis and dissertations concluded and teacher's publications. A panel data model was estimated for 35 University's education and research units, employing data for the period 1996 to 1999. Our results provided evidence of the existence of productspecific scale economies only for undergraduate and graduate teaching. Product-specific constant returns to scale were observed in the other two products. However, product-specific economies of scope emerged in all products. Therefore, the research university model, combining undergraduation, graduation and research appeared to be more cost efficient. Finally, it was interesting to note that the marginal costs estimated were: $\mathrm{R} \$ 14.23$ for undergraduate teaching, $\mathrm{R} \$ 64.79$ for graduate teaching, $\mathrm{R} \$ 24,206.96$ for thesis and dissertations concluded and $\mathrm{R} \$ 3,976.02$ for books and papers published.

\section{Introdução}

As discussões acerca das tendências mundiais em relação ao trabalho e emprego convergem no aspecto de que a inserção no mercado de trabalho será cada vez mais difícil em decorrência da progressiva competitividade nos mercados globalizados e das constantes e, não raras vezes, abruptas mutações. Esta realidade tem reflexos diretos sobre a situação e as políticas na área educacional, especialmente no que tange ao ensino superior.

Inúmeros são os desafios que atualmente se colocam para a grande maioria dos gestores de políticas públicas relacionadas ao ensino superior ao redor do mundo. Johnstone (1998) sintetizou os principais desafios que atualmente se colocam para a grande maioria dos gestores de políticas públicas relacionadas ao ensino superior ao redor do mundo nos seguintes pontos: Pressão da Sociedade por Expansão e Diversificação da Oferta, Pressão Fiscal, Demanda por maior Justificação (Accountability), Demanda por Melhoria Qualitativa e Maior Eficiência. ${ }^{1}$

Desse modo, impõem-se aos gestores de instituições públicas, privadas e de órgãos de fomento, a necessidade de também incorporar em suas decisões os resul-

\footnotetext{
${ }^{1}$ Ver Birdsall (1996) para maiores detalhes sobre o debate acerca das pressões para redução de custos e dificuldades de financiamento do ensino superior. BID (1997), Psacharopoulos (1996) e World Bank (1994) também posicionam-se a respeito destes temas. Diaz (1999) discute algumas das questões relacionadas à geração de ineficiências internas em universidades públicas, analisando determinadas posturas dos alunos dos cursos de graduação em Economia.
} 
tados de análises e estudos mais técnicos acerca das características das atividades desenvolvidas em instituições de ensino e pesquisa. Algumas das questões mais prementes são:

- as instituições estão trabalhando de forma eficiente? Haveria ganhos em aumentar a escala de produção?

- o conjunto de atividades de pesquisa pode ser produzido independentemente das atividades de ensino de graduação? E de pós-graduação? Os conjuntos podem ser produzidos separadamente de forma eficiente, ou seja, com custos mais baixos?

Reconhecendo, como já haviam feito Verry e Layard (1975) que as universidades são, na verdade, instituições que produzem múltiplos produtos, Cohn et alii (1989) estimaram uma função custo multi-produto para instituições de ensino superior, procurando mensurar o grau de economias de escala e de escopo, seguindo a metodologia proposta por Baumol, Panzar e Willig em artigo seminal de 1982. A contribuição do referido trabalho ao debate atual acerca deste tema foi marcante.

Posteriormente, vários foram os estudos dedicados à estimação de economias de escala e de escopo em instituições de ensino superior utilizando modelos com distintas variáveis e formas funcionais. Entre eles devem ser citados de de Groot et alii (1991), Glass et alii (1995b), Glass et alii (1995a), Koshal e Koshal (1995), Johnes (1997), Koshal e Koshal (1999) e Koshal et alii (2001).

No Brasil, a discussão acerca das características da função de produção/custos nas universidades é bastante incipiente, na medida em que se verificam contribuições esporádicas. Merece destaque o trabalho recente de Marinho et alii (1997), em que os autores procuram avaliar a eficiência das universidades federais brasileiras, gerando inclusive um conjunto de escores de eficiência para as universidades consideradas, por meio da técnica de análise envoltória de dados. Note-se que não há registro de publicações recentes relacionadas à estimações de funções custo e mesmo à avaliação da existência de economias de escala e de escopo em instituições de ensino superior.

É justamente para o preenchimento desta lacuna que este artigo pretende contribuir. Neste sentido, estima-se uma função custo multi-produto, cujos produtos são os seguintes: ensino de graduação, pós-graduação, títulos de pós-graduação outorgados e publicações. Utilizam-se informações referentes a 35 unidades de ensino e pesquisa da Universidade de São Paulo para o período 1996 a 1999. A partir dos resultados obtidos pode-se avaliar a existência de economias de escala e de escopo, incorporando, entretanto, a existência de diferenças sistemáticas entre as unidades de ensino e pesquisa. 


\section{Metodologia e Variáveis Utilizadas}

Tanto Cohn et alii (1989), como a grande maioria dos trabalhos que se seguiram, obtiveram suas estimativas a partir de dados das diversas unidades para um único período. Ocorre, entretanto, que ao proceder desta forma, ignoraram-se as diferenças sistemáticas não-observáveis entre as diversas instituições. A estimação de modelos adequados para dados em painel permite superar esta limitação.

Seguindo Koshal et alii (2001), Koshal e Koshal (1999), Hashimoto e Cohn (1997) e Cohn et alii (1989) será estimada a seguinte especificação para o modelo em que se ignoram as especificidades de cada unidade:

$$
C T=a_{0}+\sum_{k=1}^{4} a_{k} Q_{k}+\frac{1}{2} \sum_{k=1}^{4} \sum_{j=1}^{4} b_{k j} Q_{k} Q_{j}+\epsilon
$$

onde:

$C T$ é o Custo Total;

$Q_{k}$ são as unidades produzidas de cada um dos produtos considerados - ensino de graduação, ensino de pós-graduação, teses e publicações;

$a_{0}, a_{k}$ e $b_{k j}$ são parâmetros a serem estimados;

$\epsilon$ é o termo aleatório.

Esta especificação assume que o custo de produção de cada instituição, no caso, cada unidade da USP, pode ser representado por uma função quadrática flexível de custos fixos (flexible fixed cost quadratic function).

Ocorre, entretanto, conforme inicialmente mencionado, que nesta definição estão sendo ignoradas as diferenças sistemáticas existentes entre as unidades. Neste caso, devemos considerar que:

$$
\epsilon=\mu_{i}+v_{i t}
$$

onde:

$\mu_{i}$ representa o efeito não-observável específico de cada unidade e, $\nu_{i t}$ representa a parte restante do resíduo.

Assim, o modelo a ser analisado será:

$$
C T_{i}=\left(a_{0}+\mu_{i}\right)+\sum_{k=1}^{4} a_{k} Q_{k}+\frac{1}{2} \sum_{k=1}^{4} \sum_{j=1}^{4} b_{k j} Q_{k} Q_{j}+v
$$


A partir das estimativas obtidas por meio dos modelos apresentados, é possível mensurar as seguintes variáveis:

$$
C M I_{G R A D}=\frac{C T\left(Q_{G R A D}, Q_{P O S}, Q_{T E S E S}, Q_{P U B}\right)-C T\left(0, Q_{P O S}, Q_{T E S E S}, Q_{P U B}\right)}{Q_{G R A D}}
$$

onde:

$C M I_{G R A D}$ é o Custo Médio Incremental da Produção de Ensino de Graduação ensino com aprovação;

$C T$ é o Custo Total de produção das respectivas quantidades de cada um dos produtos, $Q_{G R A D}, Q_{P O S}, Q_{T E S E S}, Q_{P U B}$;

$Q_{G R A D}$ unidades de ensino de graduação efetivo;

$Q_{P O S}$ unidades de ensino de pós-graduação efetivo;

$Q_{T E S E S}$ unidades de títulos outorgados;

$Q_{P U B}$ unidades de publicações.

Observar que esta variável representa o custo médio de produção do produto ensino de graduação já que no numerador encontra-se a diferença entre o custo total de produção de todos os produtos descontado do custo de produção dos demais produtos com exceção do ensino de graduação. Tem-se deste modo uma estimativa do custo do ensino de graduação dividida pela quantidade produzida, resultando na estimativa do custo médio do referido produto.

$$
E_{\text {Grad }}=\frac{C M I_{G R A D}}{C M g_{G R A D}}
$$

onde $E_{G R A D}=$ economia de escala produto-específica do ensino de graduação e;

$$
C M g_{G R A D}=\frac{\partial C T}{\partial Q_{G R A D}}
$$

onde $C M g_{G R A D}$ é o Custo Marginal de produção de ensino de graduação.

Genericamente, considera-se que existem economias de escala quando o custo total de uma firma em produzir um determinado produto/serviço é menor do que o somatório do custo total de duas ou mais firmas em produzirem este mesmo produto/serviço. Se $E_{G R A D}$ for maior (menor) que a unidade, considera-se que existem economias(deseconomias) de escala produto-específica para o produto graduação. O valor unitário representa a situação de retornos constantes de escala. ${ }^{2}$

\footnotetext{
${ }^{2}$ Para compreender a variável $E_{G R A D}$ como representativa da presença de economias/deseconomias de escala no contexto de uma função multi-produto, pode-se fazer uma analogia com o tipo de análise realizada na presença de uma função de produção convencional. Neste
} 
onde $E S_{G R A D}=$ economia de escopo produto-específica do ensino de graduação.

Considera-se que existem economias de escopo em um processo produtivo quando o custo total de uma firma em produzir conjuntamente, pelo menos dois produtos/serviços, é menor do que o custo de duas ou mais firmas produzirem separadamente estes mesmos produtos/serviços, a preços dados de insumos. Na equação 7 , o termo $C T\left(Q_{G R A D}, Q_{P O S}, Q_{T E S E S}, Q_{P U B}\right)$ representa o custo de produção conjunta. Havendo economias de escopo, o custo conjunto será inferior à soma dos custos individuais, de tal modo que $E S_{G R A D}$ será maior do que 0.

A referida medida representa o grau de presença de economias de escopo, já que, essencialmente, avalia qual a porcentagem do custo da produção poderia ser economizada caso dois (ou mais) produtos fossem produzidos em conjunto em vez de individualmente. Em geral, quanto maior for o valor de $E S_{G R A D}$, maiores serão as economias de escopo. Resumindo, se $E S_{G R A D}$ for maior (menor) que zero, considera-se que existem economias (deseconomias) de escopo produto-específica para o produto graduação.

Para os demais produtos - ensino pós-graduação, títulos de pós-graduação e pesquisa (publicações) aplicam-se conceitos análogos.

A seguir são apresentadas as definições de cada variável utilizada na aplicação empírica, organizadas por categoria.

\subsection{Produção}

No caso das atividades de Ensino, em nível de graduação e de pós-graduação, a quantificação foi possível, por meio da utilização do conceito de Ensino Assistido com Aprovação. O cálculo das variáveis referentes ao Ensino Assistido com Aprovação baseia-se, essencialmente, na obtenção do total de horas que os alunos despenderam nas atividades associadas a cada uma das disciplinas em que obtiveram aprovação. Neste contexto, o aluno seria a "matéria-prima" que é transformada por outros insumos para que se atinja o produto final desejado, ou seja, o aluno com mais conhecimentos. ${ }^{3}$

caso, se, por exemplo, o custo médio for maior que o marginal, a produção adicional gerará custos adicionais menores $\left(C M g_{G R A D}\right)$ reduzindo o custo médio final - evidência da presença de economias de escala.

${ }^{3}$ Deve-se destacar, entretanto, que entre os educadores, pedagogos e psicólogos educacionais existem algumas restrições à concepção de que aprovação poderia ser tomada como indicativo 
Sendo assim, a medida de produto depende da mensuração do número de alunos que freqüentaram aulas nos cursos de graduação ou nos cursos de pósgraduação, obtendo aprovação e, do total de horas-aula e horas-atividade associadas a cada uma das referidas disciplinas. A desagregação deste indicador pode chegar até ao nível de departamento, ou mesmo área dentro de cada departamento. Para o objetivo deste estudo trabalhou-se a nível de Unidade de Ensino. Assim, temos os totais de horas-aula "aproveitadas" pelos alunos de graduação e de pósgraduação nos anos de 1996 a 1999 com desagregação por Unidade.

Formalmente:

Horas-aula efetivas $=\sum_{i=1}^{n}$ alunos aprovados [(horas_teoria+horas_prática) duração]

onde:

$n$ é o número total de disciplinas vinculadas à unidade;

alunos aprovados $=$ considera-se somente o número de alunos matriculados em disciplinas de graduação ou pós em que obtiveram aprovação;

horas_teoria e horas_prática $=$ número de horas semanais de aulas, teóricas e práticas, respectivamente, de cada disciplina;

duração = duração de cada disciplina em semanas.

É importante notar que a metodologia utilizada para a mensuração da variável Ensino Assistido com Aprovação apresenta uma grande vantagem em relação às medidas de produção convencionalmente utilizadas, que é a de atribuir a cada unidade, o total da produção gerada por seus docentes, independentemente da unidade da USP em que a disciplina tenha sido ministrada. Isto se deve ao fato de que a totalização é feita a partir das disciplinas, às quais estão associados códigos que identificam a unidade de origem do docente responsável.

Relativamente à produção de pós-graduação, será avaliado mais um aspecto, além do Ensino Assistido com Aprovação, mencionado acima. Refere-se aos títulos de pós-graduação outorgados em cada uma das unidades da USP. Com isto, incorpora-se a produção em nível de pós-graduação tanto pelo aspecto de incremento no conhecimento dos alunos obtido pela freqüência aos cursos, como a própria capacitação como pesquisador, representada pela competência para a concretização de um trabalho científico.

que o indivíduo tornou-se mais educado. Ver Coombs e Hallak (1989) para uma discussão mais profunda acerca deste debate. 
A produção referente às atividades de pesquisa foi representada pela publicação de trabalhos em periódicos e livros nacionais e internacionais. Estas informações foram retiradas do Anuário Estatístico da Universidade de São Paulo. A este respeito, merece destaque o fato de que apenas foram contabilizados os livros e os artigos publicados pelos docentes de cada uma das unidades avaliadas. ${ }^{4}$ Assim sendo, não se diferenciaram ou ponderaram as publicações por critérios de qualidade normalmente adotados, como por exemplo, número de páginas, fatores de impacto, ${ }^{5}$ porque esta avaliação exigiria o desenvolvimento de uma metodologia específica que contemplasse, por exemplo, a criação de critérios comparativos entre grandes áreas (biológicas e da saúde, exatas, humanas, etc.), e ademais, entre básicas e aplicadas. Enfim, uma tarefa que supera, e muito, o escopo deste trabalho.

De qualquer modo, deve-se destacar que desdobramentos desta linha de investigação poderão passar por aperfeiçoamentos na medida de produção de pesquisas.

\subsection{Custos}

Foram levantadas as informações acerca do custo do pessoal ativo e inativo, incluindo funcionários e docentes e o total das despesas de custeio, o que permitiu a obtenção do custo total. ${ }^{6}$ Essencialmente, esta será a variável dependente dos modelos a serem estimados.

É importante destacar, no entanto, os critérios adotados para a incorporação dos custos da Administração Central. Utilizaram-se dois métodos, justamente para analisar a sensibilidade dos resultados a variações neste tipo de procedimento. O primeiro, correspondeu à apropriação dos referidos custos entre as diversas unidades de ensino e pesquisa.

$\mathrm{Na}$ tabela 1 estão relacionados os órgãos centrais que possuem uma contabilização de gastos em separado, cujos valores foram redistribuídos pelas diversas

\footnotetext{
${ }^{4}$ Em consulta feita ao setor responsável pela elaboração deste levantamento, verificou-se que no caso de trabalhos com autores pertencentes a distintas unidades pode, eventualmente, existir um problema de dupla contagem na referida informação. Em uma tentativa de quantificar a magnitude deste desvio, foi solicitado um levantamento sobre o total de publicações de artigos em periódicos e livros da Universidade, sem a distinção por unidades. Comparou-se este valor com aquele obtido a partir da totalização gerada a partir das informações desagregadas por unidades. Verificou-se uma diferença média no período que oscilou em torno dos 8\%, nível considerado aceitável para os objetivos deste trabalho.

${ }^{5}$ Uma importante contribuição nesta direção, apenas para a área de Economia, é Issler e Pillar (2002).

${ }^{6}$ Tendo em vista a atual estrutura de custos das universidades públicas, pode-se considerar que as despesas com inativos correspondem à mais importante parcela dos custos fixos.
} 
unidades de ensino.

Tabela 1

Relação dos órgãos centrais de direção e serviço e das unidades para as quais foram redistribuídos os respectivos custos

\begin{tabular}{|c|c|c|}
\hline Sigla & Nome da Unidade & Distribuição para: \\
\hline RUSP & Reitoria & Todas as unidades \\
\hline COSEAS & Coordenadoria de Assistência Social & Todas as unidades \\
\hline FUNDUSP & Fundo de Construção da USP - FUNDUSP & Todas as unidades \\
\hline CCS & Coordenadoria de Comunicação Social & Todas as unidades \\
\hline $\mathrm{CCE}$ & Centro de Computação Eletrônica & Todas as unidades \\
\hline $\mathrm{PCO}$ & Prefeitura da Cidade Universitária & $\begin{array}{l}\text { Todas as unidades excluindo-se aquelas } \\
\text { que não se localizam dentro do } \\
\text { campus Armando de Salles Oliveira } \\
\text { (São Paulo) e as unidades do interior }\end{array}$ \\
\hline CEPEUSP & do interior. Centro de Práticas Esportivas & $\begin{array}{l}\text { Todas as unidades excluindo-se } \\
\text { do interior }\end{array}$ \\
\hline PCAB & Pref do Campus de Bauru & Faculdade de Odontologia de Bauru \\
\hline PCAPS & Prefeitura do Campus de Pirassununga & $\begin{array}{l}\text { Somente unidades situadas no campus } \\
\text { de Pirassununga }\end{array}$ \\
\hline PCASC & Pref do Campus Adm de São Carlos & $\begin{array}{l}\text { Somente unidades situadas no campus } \\
\text { de São Carlos }\end{array}$ \\
\hline CISC & Centro de Informática de São Carlos & $\begin{array}{l}\text { Somente unidades situadas no campus } \\
\text { de São Carlos }\end{array}$ \\
\hline PCARP & Pref do Campus de Rib Preto & $\begin{array}{l}\text { Somente unidades situadas no campus } \\
\text { de Ribeirão Preto }\end{array}$ \\
\hline PCLQ & Pref do Campus Luiz de Queiroz & $\begin{array}{l}\text { Escola Superior de Agricultura } \\
\text { "Luiz de Queiroz" }\end{array}$ \\
\hline
\end{tabular}

A seleção das unidades para as quais iriam os recursos da redistribuição foi feita com base em hipóteses sobre a amplitude da atuação de cada um dos órgãos. Assim, as prefeituras dos campi do interior têm sua atuação limitada à cidade onde se localizam. Com isso, seus custos foram distribuídos apenas para as unidades que ali se localizam. Os custos da Reitoria, COSEAS, FUNDUSP, CCS e CCE foram redistribuídos entre todas as unidades pois supôs-se que realizam trabalhos que atendem toda a Universidade. Já o CEPEUSP dificilmente poderá beneficiar 
alunos de escolas do interior, mas certamente sua utilização é ampla entre os alunos moradores da capital, independentemente de estudarem em unidades situadas ou não no campus da Cidade Universitária "Armando de Salles Oliveira". Assim, alunos das Faculdades de Direito e Medicina, por exemplo, não teriam dificuldades extras para a utilização do CEPEUSP.

A apropriação dos organismos que devem se distribuir entre todas as unidades da USP, ou seja, RUSP, COSEAS, FUNDUSP, CCS e CCE foi realizada com base na proporção que cada unidade de ensino e pesquisa possuía no Custeio Total, ou seja, despesas com pessoal ativo, material de consumo e serviços de terceiros. A utilização deste critério partiu do pressuposto de que unidades que demandam mais recursos de custeio provavelmente demandarão mais recursos destes órgãos centrais. Os demais organismos tiveram suas despesas rateadas em parcelas iguais entre as diversas unidades em decorrência das características dos serviços prestados tornarem menos adequada a hipótese adotada anteriormente.

Justamente em decorrência das dificuldades envolvidas nesta tarefa de apropriação, optou-se também por trabalhar com um segundo critério, em que os custos dos organismos centrais foram excluídos da análise. Conforme mencionado anteriormente, esta opção tem por objetivo analisar a sensibilidade das estimativas obtidas ao tipo de procedimento adotado para o tratamento dos custos da administração central da universidade.

É preciso destacar que na construção das variáveis de custo foram utilizadas as informações acerca das despesas empenhadas, pois considera-se que melhor representam o custo incorrido na produção dos bens/serviços.

Finalmente, um último ponto a considerar refere-se ao fato de que está sendo assumido que os preços dos fatores produtivos não variam entre as distintas unidades da USP, tendo em vista a política de isonomia salarial adotadas entre as três universidades estaduais paulistas. Também, reforça tal hipótese o fato do período analisado (1996 a 1999) ser relativamente curto, não se verificando mudanças de preços relativos que permitissem a incorporação de variáveis relacionadas aos mesmos nos modelos. Obviamente, um painel mais longo em termos da série temporal analisada, exigiria uma análise mais detalhada acerca da necessidade de inclusão destes componentes. ${ }^{7}$

\footnotetext{
${ }^{7}$ Maiores detalhes podem ser encontrados em Koshal et alii (2001) e Hashimoto e Cohn (1997).
} 


\section{Resultados}

Inicialmente, deve-se destacar que cada um dos modelos foi estimado em duplicidade, em decorrência da opção metodológica, mencionada anteriormente, de trabalhar com os custos totais incorporando os custos dos organismos de administração central e não incorporando os referidos custos administrativos. Assim, para cada especificação haverá um modelo estimado tendo como variável explicada o custo total incluindo custos centrais, que será denominado modelo 1 que é o modelo completo, e um outro, tendo como variável explicada apenas o custo total de cada uma das unidades de ensino, sem qualquer apropriação dos custos administrativos dos organismos centrais, que será chamado modelo 2.

Optou-se, a princípio, por estimar um modelo como tradicionalmente produzido na literatura sobre o tema, ou seja, pelo método de mínimos quadrados, seguindo, principalmente, Koshal e Koshal (1999) e Cohn et alii (1989) e que ignora as diferenças sistemáticas não-observáveis entre as diversas unidades.

Na tabela 2 encontram-se a definição e algumas medidas estatísticas básicas das variáveis consideradas.

Tabela 2

Definição das variáveis e estatísticas básicas

\begin{tabular}{|c|c|c|c|c|c|}
\hline Variável & Descrição das variáveis & Média & Desvio padrão & Min & Max \\
\hline custo_total & $\begin{array}{l}\text { custo total por unidade da USP, incluindo } \\
\text { apropriação da Adm. Central } \\
\text { (em milhões de reais/ano) }\end{array}$ & 20,9 & 12,8 & 5,1 & 56,8 \\
\hline custo_unidade & $\begin{array}{l}\text { custo total por unidade da USP, excluindo } \\
\text { Adm. Central (em milhões de reais/ano) }\end{array}$ & 16,2 & 10,6 & 2,3 & 49,9 \\
\hline gradapr & $\begin{array}{l}\text { produção graduação: horas-aula que } \\
\text { geraram aprovação (em milhares/ano) }\end{array}$ & 584,6 & 554,2 & 17,0 & $2.291,8$ \\
\hline posapr & $\begin{array}{l}\text { produção pós-graduação: horas-aula que } \\
\text { geraram aprovação (em milhares/ano) }\end{array}$ & 47,1 & 39,3 & 3,0 & 229,0 \\
\hline postes & $\begin{array}{l}\text { produção pós-graduação: número de títulos } \\
\text { outorgados (em milhares/ano) }\end{array}$ & 0,1 & 0,1 & 0,0 & 0,4 \\
\hline public & $\begin{array}{l}\text { produção pesquisa: } \\
\text { publicações (em milhares/ ano) }\end{array}$ & 0,2 & 0,2 & 0,0 & 1,1 \\
\hline gradapr2 & $\begin{array}{l}\text { graduação: horas-aula que geraram } \\
\text { aprovação (em milhares/ano) }\end{array}$ & $646.700,5$ & $1.136 .286,0$ & 289,9 & $5.252 .439,0$ \\
\hline posapr 2 & pós-graduação: posapr ${ }^{2}$ & $3.748,2$ & $6.736,0$ & 8,8 & $52.426,4$ \\
\hline
\end{tabular}




\begin{tabular}{|c|c|c|c|c|c|}
\hline Variável & Descrição das variáveis & Média & Desvio padrão & Min & Max \\
\hline postes 2 & pós-graduação: postes ${ }^{2}$ & 0,0 & 0,0 & 0,0 & 0,2 \\
\hline public2 & pesquisa: public ${ }^{2}$ & 0,1 & 0,2 & 0,0 & 1,1 \\
\hline gradposapr & gradapr $\mathrm{x}$ posapr & $42.679,5$ & $73.780,7$ & 242,5 & $524.753,4$ \\
\hline gradaprpostes & gradapr $\mathrm{x}$ postes & 91,0 & 178,7 & 0,2 & 944,2 \\
\hline gradaprpublic & gradapr $\mathrm{x}$ public & 199,7 & 372,2 & 0,4 & $2.069,7$ \\
\hline postespublic & postes $\mathrm{x}$ public & 0,0 & 0,1 & 0,0 & 0,4 \\
\hline posaprpublic & posapr $\mathrm{x}$ public & 14,6 & 26,8 & 0,1 & 200,8 \\
\hline
\end{tabular}

Os resultados da estimação dos modelos preliminares encontram-se na tabela 3:

Tabela 3

Estimação do modelo preliminar por mínimos quadrados

\begin{tabular}{|c|c|c|c|c|c|c|}
\hline & \multicolumn{3}{|c|}{ Modelo 1} & \multicolumn{3}{|c|}{ Modelo 2} \\
\hline Variável & Coef. & $t$ & $P>|t|$ & Coef. & $t$ & $P>|t|$ \\
\hline gradapr & 0,0136 & 4,0300 & 0,0000 & 0,0079 & 2,9400 & 0,0040 \\
\hline posapr & 0,1023 & 2,1900 & 0,0300 & 0,0857 & 2,3100 & 0,0220 \\
\hline postes & 72,6729 & 2,7900 & 0,0060 & 64,7322 & 3,1300 & 0,0020 \\
\hline public & 21,0117 & 1,8900 & 0,0610 & 19,4233 & 2,2000 & 0,0290 \\
\hline gradapr 2 & 0,0000 & $-1,5700$ & 0,1190 & 0,0000 & $-1,7100$ & 0,0900 \\
\hline posapr2 & $-0,0009$ & $-1,8200$ & 0,0710 & $-0,0007$ & $-1,8600$ & 0,0650 \\
\hline postes 2 & $-178,2305$ & $-1,6500$ & 0,1020 & $-163,8754$ & $-1,9100$ & 0,0590 \\
\hline public2 & 13,1899 & 0,7900 & 0,4280 & $-5,9577$ & $-0,4500$ & 0,6520 \\
\hline gradposapr & 0,0000 & 0,0300 & 0,9770 & 0,0000 & 0,8600 & 0,3930 \\
\hline gradaprpos & 0,0597 & 1,8000 & 0,0740 & 0,0408 & 1,5500 & 0,1240 \\
\hline gradaprpub & $-0,0388$ & $-3,4400$ & 0,0010 & $-0,0189$ & $-2,1100$ & 0,0370 \\
\hline postespubl & 22,7204 & 0,2500 & 0,8050 & 42,4013 & 0,5800 & 0,5610 \\
\hline posaprpubl & 0,2959 & 1,7500 & 0,0820 & 0,1214 & 0,9000 & 0,3680 \\
\hline cons & 2,6316 & 2,6900 & 0,0080 & 1,4299 & 1,8400 & 0,0690 \\
\hline & $\begin{array}{c}\text { Númerc } \\
\mathrm{F}(13,126) \\
\mathrm{R} 2=0,8\end{array}$ & $\begin{array}{l}\text { o de Obser } \\
=81,83 \mathrm{P} \\
941 \mathrm{R} 2 \mathrm{aju}\end{array}$ & $\begin{array}{l}\text { es }=140 \\
F=0,0000 \\
\text { lo }=0,8832\end{array}$ & $\begin{array}{c}\text { Númerc } \\
\mathrm{F}(13,126) \\
\mathrm{R} 2=0,9\end{array}$ & $\begin{array}{l}\text { de Obse } \\
=90,02 \mathrm{P} \\
028 \mathrm{R} 2 \mathrm{aj}\end{array}$ & $\begin{array}{l}\text { es }=140 \\
F=0,0000 \\
o=0,8928\end{array}$ \\
\hline
\end{tabular}

A partir deste modelo foram estimadas as medidas de economias de escala e de escopo, conforme definidas nas equações 5 e 7 e que podem ser vistas na tabela 4: 
Tabela 4

Economias de escala e de escopo produto-específicas $\left(^{*}\right)$ método de mínimos quadrados ordinários

\begin{tabular}{lcccc}
\hline & $\begin{array}{c}\text { Ensino } \\
\text { Graduação }\end{array}$ & $\begin{array}{c}\text { Ensino } \\
\text { Pós Graduação }\end{array}$ & $\begin{array}{c}\text { Títulos de } \\
\text { Pós Graduação }\end{array}$ & Publicações \\
\hline & \multicolumn{3}{c}{ Modelo 1 } & \\
\hline Economias de Escala (E) & 1,59 & 1,53 & 1,21 & 0,86 \\
Economias de Escopo (Es) & 0,18 & $-0,02$ & $-0,04$ & 0,17 \\
\hline & & Modelo 2 & \\
\hline & 1,58 & 1,53 & 1,23 & 1,08 \\
Economias de Escala (E) & 0,03 & $-0,05$ & $-0,09$ & 0,09 \\
\hline
\end{tabular}

$\left(^{*}\right)$ calculadas no valor médio de cada uma das variáveis relevantes

Estes resultados indicam, a princípio que, pelo menos, em termos das medidas obtidas a partir dos modelos lineares, o método de apropriação dos custos da administração central não provoca grandes distorções nos resultados das medidas obtidas. A exceção é a medida de economias de escala produto-específica da produção de pesquisas (publicações), em que a utilização do modelo 1 indica a presença de deseconomias de escala enquanto os resultados obtidos a partir do modelo 2 indicam a presença de economias de escala. Para os demais produtos ambos os modelos indicaram a presença de economias de escala produto-específicas.

Em relação às economias de escopo, as indicações foram consistentes entre os dois modelos. Foram encontradas economias de escopo produto-específicas para as atividades de ensino de graduação e publicações. No caso, dos dois produtos vinculados à pós-graduação as indicações não são muito claras, já que os coeficientes obtidos a partir do Modelo 1, apesar de negativos, indicativo de deseconomias de escopo, encontram-se relativamente próximos de zero. Os resultados do Modelo 2, por outro lado, sinalizam a presença de deseconomias de escopo para os produtos associados à pós-graduação.

Porém, tendo em vista a diversidade das instituições avaliadas, torna-se essencial investigar a hipótese de existência de diferenças sistemáticas não-observáveis entre elas. Isto será possível por meio da estimação do modelo para dados em painel.

Inicialmente, optou-se por estimar o modelo para dados em painel que considera os efeitos aleatórios, cujos resultados encontram-se na tabela 5. Somente 
a partir deste modelo é possível aplicar testes essenciais para uma melhor especificação do modelo. Duas são as hipóteses a serem testadas: de existência de efeitos individuais (Teste Breusch Pagan), que permite concluir pela adequação ou não, do modelo linear convencional, e da ortogonalidade dos efeitos (Teste de Hausman), crucial no modelo de efeitos aleatórios.

O teste Breusch Pagan é um teste do tipo Lagrange Multiplier, cuja hipótese nula é $\operatorname{Var}\left(\mu_{i}\right)=0$ e a alternativa é $\operatorname{Var}\left(\mu_{i}\right) \neq 0$. A rejeição da hipótese nula leva à conclusão da existência de efeitos individuais, e a inadequação do modelo estimado por mínimos quadrados com uma única constante ( $a_{0}$ na equação 1 ).

O Hausman é um teste do tipo Wald, cuja hipótese nula é que as diferenças nos coeficientes não são sistemáticas, ou seja, os coeficientes do modelo e os efeitos aleatórios são ortogonais. A rejeição da hipótese nula indica que a melhor escolha é o modelo de efeitos fixos, tendo em vista que esta condição é essencial no modelo de efeitos aleatórios. Maiores detalhes podem ser encontrados em Greene (2000).

Tabela 5

Estimação do modelo efeitos aleatórios

\begin{tabular}{|c|c|c|c|c|c|c|}
\hline \multirow[b]{2}{*}{ Variável } & \multicolumn{3}{|c|}{ Modelo 1} & \multicolumn{3}{|c|}{ Modelo 2} \\
\hline & Coef. & $z$ & $P>|z|$ & Coef. & $z$ & $P>|z|$ \\
\hline gradapr & 0,0158 & 6,4200 & 0,0000 & 0,0134 & 5,9200 & 0,0000 \\
\hline posapr & 0,0725 & 3,4100 & 0,0010 & 0,0742 & 3,7400 & 0,0000 \\
\hline postes & 24,6591 & 2,9200 & 0,0040 & 32,8712 & 4,1300 & 0,0000 \\
\hline public & 3,7682 & 0,9300 & 0,3540 & 4,9854 & 1,3000 & 0,1950 \\
\hline gradapr2 & 0,0000 & $-3,2300$ & 0,0010 & 0,0000 & $-3,2900$ & 0,0010 \\
\hline posapr 2 & $-0,0006$ & $-4,0800$ & 0,0000 & $-0,0006$ & $-4,2400$ & 0,0000 \\
\hline postes 2 & $-19,5037$ & $-0,5100$ & 0,6120 & $-34,4626$ & $-0,9500$ & 0,3420 \\
\hline public2 & 1,1341 & 0,2500 & 0,7990 & $-1,7112$ & $-0,4000$ & 0,6860 \\
\hline gradposapr & 0,0000 & 2,5100 & 0,0120 & 0,0000 & 3,4300 & 0,0010 \\
\hline gradaprpostes & 0,0144 & 1,2300 & 0,2190 & 0,0144 & 1,3100 & 0,1900 \\
\hline gradaprpublic & $-0,0042$ & $-1,1500$ & 0,2490 & $-0,0018$ & $-0,5000$ & 0,6140 \\
\hline postespublic & $-26,8460$ & $-1,2800$ & 0,2000 & $-26,2951$ & $-1,3200$ & 0,1860 \\
\hline posaprpublic & 0,1040 & 2,1200 & 0,0340 & 0,0796 & 1,7100 & 0,0870 \\
\hline _cons & 7,9702 & 6,3600 & 0,0000 & 3,2791 & 3,1200 & 0,0020 \\
\hline
\end{tabular}




\begin{tabular}{|c|c|c|c|c|c|c|c|}
\hline & \multicolumn{4}{|c|}{ Modelo 1} & \multicolumn{3}{|c|}{ Modelo 2} \\
\hline Variável & Coef. & $z$ & $P>1$ & $|z|$ & Coef. & $z$ & $P>|z|$ \\
\hline sigma_u & & 4,6356 & & & & 3,8689 & \\
\hline sigma_e & & 0,7197 & & & & 0,7376 & \\
\hline $\begin{array}{l}\text { rho (fração de } \\
\text { variância devida a } u_{i} \text { ) }\end{array}$ & & 0,9765 & & & & 0,9649 & \\
\hline
\end{tabular}

A estatística do teste Breusch and Pagan obtida a partir do Modelo 1 foi de chi2 $(1)=129,06$, sendo que a Prob $>$ chi $2=0,0000$, o que leva à rejeição da hipótese nula. Para este modelo, a estatística do teste de especificação de Hausman foi de chi2(13) =0,00 com Prob $>$ chi2 $=0,9999$, levando, por outro lado, à não rejeição da hipótese nula. Sendo assim, conclui-se que o modelo linear convencional não é adequado e a melhor opção é o modelo com efeitos aleatórios, cujos resultados encontram-se em destaque.

No Modelo 2, a estatística do teste Breusch and Pagan foi de chi2(1) = 149,70, sendo que a Prob $>$ chi2 $=0,0000$, o que leva à rejeição da hipótese nula. Neste caso, a estatística do teste de especificação de Hausman foi de chi2(13) = 20,69 com Prob $>$ chi $2=0,0794$, o que leva, a uma certa dúvida quanto à decisão. Porém, considerando o objetivo inicial de verificar a sensibilidade dos resultados à utilização de distintas maneiras de incorporar os gastos da administração central e utilizando um nível de significância de 10\%, concluimos pela rejeição da hipótese nula. Sendo assim, consideramos que o modelo linear convencional não é adequado e a melhor opção, neste caso, é o modelo com efeitos fixos.

Para seguir com a estratégia da comparabilidade, foram estimados os modelos com efeitos fixos para as duas especificações, ressaltando-se, porém, que serão utilizados apenas os resultados referentes ao modelo 2, já que para o modelo 1, a especificação que mostrou-se mais adequada, de acordo com os testes de especificação, foi a de efeitos aleatórios. Na tabela 6 encontram-se os resultados das estimativas dos Modelos de Efeitos Fixos, e em destaque as estimativas do modelo 2 . 
Tabela 6

Estimação do modelo efeitos fixos

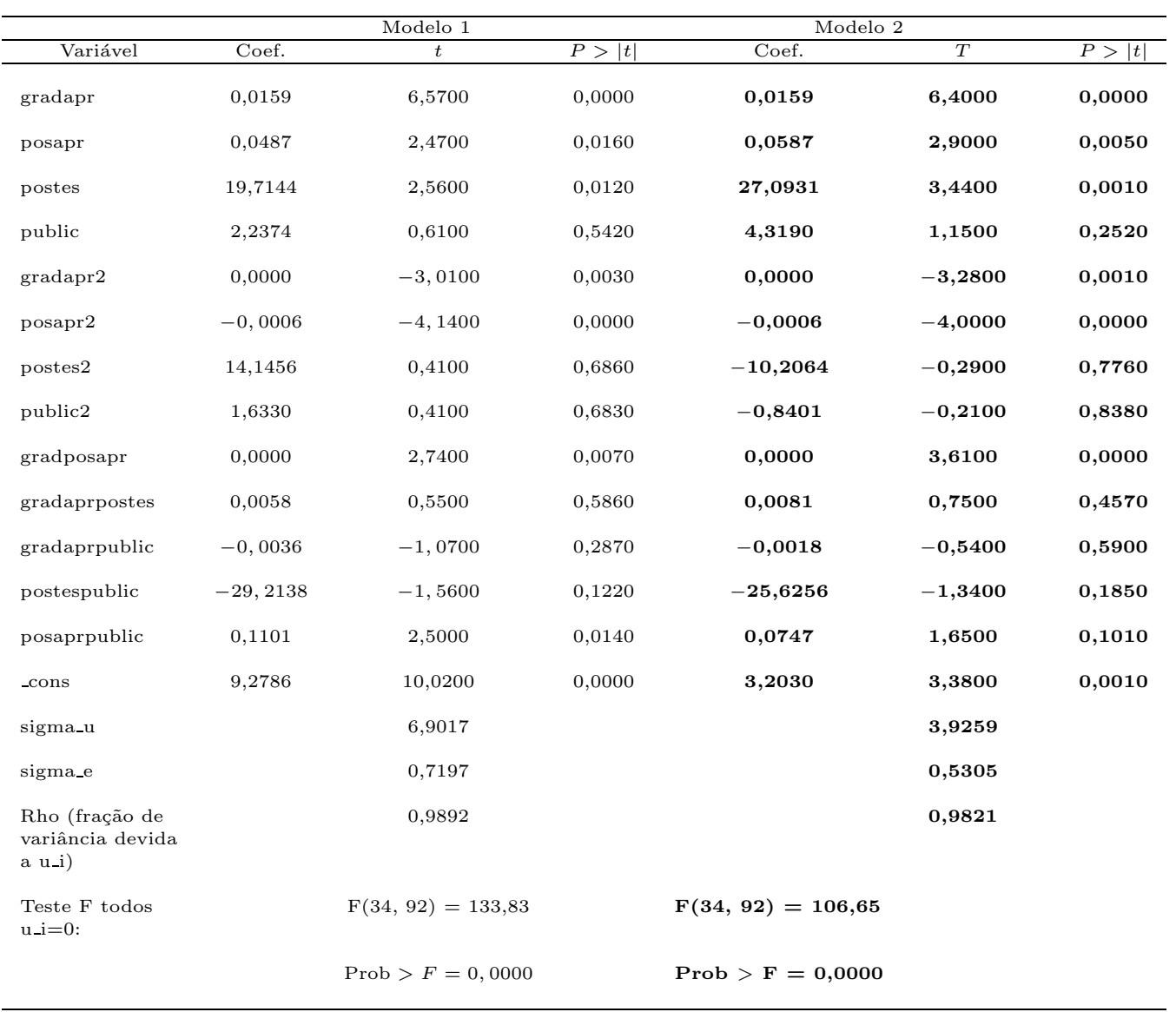

Considerando os resultados das duas especificações mais adequadas, ou seja, a de efeitos aleatórios para o Modelo 1 e a de Efeitos Fixos para o Modelo 2, deve-se destacar que os coeficientes dos termos de interação entre as variáveis de produto (gradposapr, gradaprpostes, gradaprpublic, postespublic, posaprpublic) correspondem aos produtos marginais cruzados. Deste modo, um coeficiente negativo implica complementaridade de custos e positivo, substitutibilidade. Em relação ao Modelo 1 (efeitos aleatórios), verificou-se que apenas os coeficientes de duas variáveis mostraram-se estatisticamente significativos ao nível de significância de 10\% - gradposapr e posaprpublic - e, ambos com sinal positivo, evidenciando a substitutibilidade entre as atividades de ensino de graduação e de pós-graduação, e entre a produção de títulos de pós-graduação e de publicações. 
Já os resultados do modelo alternativo (Modelo 2) também sinalizam a mesma direção, mencionando-se, entretanto, que o coeficiente da variável posaprpublic não se mostrou estatisticamente significativa ao nível de $10 \%$.

Outro importante resultado que pode ser obtido a partir das estimativas acima refere-se ao custo marginal de cada produto, calculado no valor médio de cada uma das variáveis relevantes. Verifica-se na tabela 7 que o custo marginal de produção da atividade de ensino de graduação é menos de $30 \%-\mathrm{R} \$ 14,23$ pelo Modelo 1 ou $\mathrm{R} \$ 13,39$ pelo Modelo 2 - da referida medida correspondente ao nível de pós-graduação, $\mathrm{R} \$ 64,79$ e $\mathrm{R} \$ 49,89$, nos Modelo 1 e 2, respectivamente. Deve-se lembrar que estas variáveis estão medidas em horas que geraram aprovação.

Relativamente, aos demais produtos, títulos de pós outorgados e número de publicações, é interessante verificar que o custo marginal da produção de dissertações e teses é consideravelmente superior, mais de seis vezes, ao custo marginal da produção de trabalhos em periódicos e livros nacionais e internacionais.

Tabela 7

Estimativa do custo marginal (em reais)

\begin{tabular}{ccccc}
\hline Produto & Graduação & Pós-aprovação & Pós Teses & Pesquisa \\
\hline $\begin{array}{c}\text { Modelo 1: } \\
\text { Custo Marginal }\end{array}$ & 14,23 & 64,79 & $24.206,96$ & $3.976,02$ \\
\hline $\begin{array}{c}\text { Modelo 2: } \\
\text { Custo Marginal }\end{array}$ & 13,39 & 49,89 & $24.494,74$ & $4.037,86$ \\
\hline
\end{tabular}

Tabela 8

Economias de escala e de escopo produto-específicas $\left(^{*}\right)$

\begin{tabular}{lcccc}
\hline & $\begin{array}{c}\text { Ensino } \\
\text { Graduação }\end{array}$ & $\begin{array}{c}\text { Ensino } \\
\text { Pós Graduação }\end{array}$ & $\begin{array}{c}\text { Títulos de } \\
\text { Pós Graduação }\end{array}$ & Publicações \\
\hline & & Modelo 1 & & \\
\hline Economias de Escala(E) & 1,09 & 1,23 & 1,04 & 0,97 \\
Economias de Escopo(Es) & 0,32 & 0,30 & 0,34 & 0,35 \\
\hline
\end{tabular}

Modelo 2

\begin{tabular}{lllll}
\hline Economias de Escala(E) & 1,19 & 1,53 & 1,04 & 1,04 \\
Economias de Escopo(Es) & 0,09 & 0,06 & 0,19 & 0,18 \\
\hline
\end{tabular}

$\left(^{*}\right)$ calculadas no valor médio de cada uma das variáveis relevantes

Relativamente à presença de economias de escala e de escopo produto-específicas, os resultados apresentados na tabela 8 indicam mais claramente a presença de economias de escala produto-específicas para os produtos ensino de graduação 
e de pós-graduação. No caso da produção de teses e dissertações e de publicações, como as estimativas encontram-se próximas à unidade, pode-se, também, considerar a possibilidade de existência de retornos constante de escala. Os resultados evidenciam de maneira contundente, distintamente do verificado a partir dos modelos preliminares, a existência de economias de escopo produto-específicas para todas as atividades.

A esta altura, é importante notar que a adoção de distintos critérios de apropriação dos custos da administração central da USP, apesar de implicar em distinções em termos dos métodos de estimação aplicados, não produziu impactos significativos sobre os resultados finais, ou seja, sobre as medidas de economias de escala e de escopo. Deste modo, para o restante da análise serão utilizadas apenas as estimativas produzidas a partir do modelo 1 , ou seja, aquele em que se redistribuíram os custos dos órgão administrativos para cada uma das unidades de ensino.

Conforme mencionado anteriormente, o modelo final estimado, incorpora as diferenças sistemáticas existentes entre as unidades. Estas diferenças refletem-se, de acordo com a especificação utilizada, em distintas medidas de Economias de Escopo.

Os resultados das médias das medidas de Economias de Escopo ProdutoEspecífica por áreas ${ }^{8}$ encontram-se na tabela 9. Optou-se por apresentar a Faculdade de Direito isoladamente e não dentro da área de Ciências Humanas em decorrência dos resultados discrepantes em relação ao padrão observado para as demais áreas. Esta foi a única unidade a apresentar deseconomias de escopo relativamente a todos os produtos, enquanto nas demais áreas verificou-se a presença de economias de escopo relativamente a todos os produtos.

\footnotetext{
${ }^{8} \mathrm{Na}$ área de Ciências Humanas e Pedagogia foram agrupadas a Escola de Comunicações e Artes, as Faculdades de Educação, de Filosofia, Letras e Ciências Humanas do campus São Paulo, a Faculdade de Filosofia, Ciências, Letras de Ribeirão Preto e a Faculdade de Economia, Administração e Contabilidade. A área de Ciências Exatas e Engenharia reune a Escola Politécnica, a Faculdade de Arquitetura e Urbanismo, a Escola de Engenharia de São Carlos, Instituto de Física, o Instituto de Matemática e Estatística, Instituto de Química, Instituto de Ciências Matemáticas e de Computação de São Carlos, Instituto de Química de S. Carlos e o Instituto de Física de São Carlos. A área de Ciências Biológicas e da Saúde é composta pela Faculdade de Medicina, Faculdade de Saúde Pública, Escola de Enfermagem, Faculdade de Ciências Farmacêuticas dos campi de São Paulo e de Ribeirão Preto, Faculdade de Medicina de Ribeirão Preto, Escola de Enfermagem de Ribeirão Preto, pelas Faculdades de Odontologia dos campi São Paulo, Ribeirão Preto e Bauru, Escola de Educação Física e do Esporte, Instituto de Biociências, Instituto de Ciências Biomédicas, Instituto de Psicologia. Finalmente, uma área final, denominada Outras, agrupa a Faculdade de Medicina Veterinária e Zootecnia, a Escola Superior de Agricultura Luiz de Queiroz, Instituto Agronômico e Geofísico, Instituto Oceanográfico, Instituto de Geociências e a Faculdade de Zootecnia e Engenharia de Alimentos.
} 
Tabela 9

Média das economias de escopo produto-específicas por área - modelo final

\begin{tabular}{lcccr}
\hline \multicolumn{1}{c}{ Área } & $\begin{array}{c}\text { Ensino } \\
\text { Graduação }\end{array}$ & $\begin{array}{c}\text { Ensino } \\
\text { Pós Graduação }\end{array}$ & $\begin{array}{c}\text { Títulos de } \\
\text { Pós Graduação }\end{array}$ & Publicações \\
\hline Ciências Biológicas e da Saúde & 0,30 & 0,28 & 0,32 & 0,33 \\
Ciências Exatas e Engenharia & 0,25 & 0,24 & 0,28 & 0,28 \\
Ciências Humanas e Pedagogia & 0,27 & 0,25 & 0,29 & 0,30 \\
Direito & $-1,23$ & $-1,29$ & $-1,16$ & $-1,14$ \\
Outras & 0,34 & 0,33 & 0,37 & 0,37 \\
\hline
\end{tabular}

\section{Considerações Finais}

Inicialmente, convém comparar os resultados aqui obtidos com aqueles apresentados em trabalhos recentes e com alguma semelhança na função custo estimada, cujos valores encontram-se na tabela 10 .

Tabela 10

Medidas das economias de escala: comparações

\begin{tabular}{lcccc}
\hline \multicolumn{1}{c}{ Trabalho } & $\begin{array}{c}\text { Ensino } \\
\text { Graduação }\end{array}$ & $\begin{array}{c}\text { Ensino } \\
\text { Pós Graduação }\end{array}$ & $\begin{array}{c}\text { Títulos de } \\
\text { Pós Graduação }\end{array}$ & Pesquisa \\
\hline $\begin{array}{l}\text { Cohn et alii (1989) - } \\
\text { instituições públicas }\end{array}$ & 0,944 & 1,685 & - & 1,273 \\
$\begin{array}{l}\text { Hashimoto e Cohn (1997) } \\
\text { Koshal e Koshal (1999) }\end{array}$ & 0,94 & 1,01 & - & 1,12 \\
$\begin{array}{l}\text { instituições públicas } \\
\text { Koshal et alii (2001) }\end{array}$ & 1,12 & 1,39 & - & 1,00 \\
Este estudo - modelo final & 1,05 & 1,03 & - & - \\
\hline
\end{tabular}

Relativamente ao produto Ensino de Graduação deve-se ressaltar que no caso da Universidade de São Paulo assim como na avaliação realizada por Koshal e Koshal (1999), também de instituições públicas, e em Koshal et alii (2001), relativo à análise de instituições religiosas, o valor encontrado é superior à unidade, representativo da existência de economias de escala. Nos outros dois trabalhos, há indicações da presença de deseconomias de escala.

Por outro lado, em relação ao produto Ensino de Pós-Graduação, identificou-se claramente a presença de economias de escala em três dos cinco estudos, sendo que em Hashimoto e Cohn (1997) e Koshal et alii (2001), as indicações não são tão evidentes, já que as medidas encontradas estão muito próximas da unidade, permitindo que se cogite acerca da existência de retornos constantes.

Assim como em Koshal e Koshal (1999), no caso brasileiro também não foram encontradas economias de escala em relação ao produto Pesquisa, representada pelas Publicações. 
É possível também realizar comparação similar relativamente às medidas de economias de escopo produto-específicas. Os valores encontrados em estudos equivalentes estão sintetizados na tabela 11.

Tabela 11

Medidas das economias de escopo: comparações

\begin{tabular}{lcccc}
\hline \multicolumn{1}{c}{ Trabalho } & $\begin{array}{c}\text { Ensino } \\
\text { Graduação }\end{array}$ & $\begin{array}{c}\text { Ensino } \\
\text { Pós Graduação }\end{array}$ & $\begin{array}{c}\text { Títulos de } \\
\text { Pós Graduação }\end{array}$ & Pesquisa \\
\hline $\begin{array}{l}\text { Hashimoto e Cohn(1997) } \\
\text { Koshal e Koshal(1999) }\end{array}$ & 0,14 & 0,11 & - & 0,16 \\
instituições públicas & 0,05 & 0,07 & - & 0,15 \\
Koshal et al.(2001) & 0,41 & 0,41 & - & - \\
Este estudo - Modelo 1 & 0,32 & 0,30 & 0,34 & 0,35 \\
\hline
\end{tabular}

No caso da existência de economias de escopo, é interessante notar que no caso da USP, assim como nos demais trabalhos, foram identificadas em todos produtos analisados, indicando que o modelo de universidade de pesquisa, em que se produzem conjuntamente ensino e pesquisa, também é o mais eficiente em termos de custos.

Finalmente, este trabalho evidenciou a possibilidade de se avaliar a estrutura de custos de unidades de uma mesma instituição de ensino superior de forma a produzir informações que possibilitem aos gestores tomar decisões que resultem em um melhor aproveitamento dos recursos disponíveis e em uma maior eficiência na alocação dos mesmos.

\section{Referências}

BID (1997). La educación superior en América Latina y el Caribe. Documento de Estratégia, Dezembro.

Birdsall, N. (1996). Public spending on higher education in developing countries: Too much or too little? Economics of Education Review, 15(4):407-419.

Carey, K. (1997). A panel data design for estimation of hospital cost functions. The Review of Economics and Statistics, 79(3):443-453.

Cohn, E., Rhine, S. L. W., \& Santos, M. C. (1989). Institutions of higher education as multi-product firms: Economies of scale and scope. The Review of Economics and Statistics, 71(2):284-290.

Coombs, P. H. \& Hallak, J. (1989). Cost Analysis in Education: A Tool for Policy and Planning. Johns Hopkins University Press. 
de Groot, H., McMahon, W. W., \& Volkwein, J. F. (1991). The cost structure of American research universities. The Review of Economics and Statistics, $73(3): 424-431$.

Diaz, M. D. M. (1999). Extended stay at university: An application of multinomial logit and duration models. Applied Economics, 31(11):1411-1422.

Glass, J. C., McKillop, D. G., \& Hyndman, N. (1995a). The achievement of scale efficiency in UK universities: A multiple-input multiple-output analysis. Education Economics, 3(3):249-263.

Glass, J. C., McKillop, D. G., \& Hyndman, N. (1995b). Efficiency in the provision of university teaching and research: An empirical analysis of UK universities. Journal of Applied Econometrics, 10:61-72.

Greene, W. H. (2000). Econometric Analysis. Prentice-Hall, Inc, New Jersey, 4th edition.

Hashimoto, K. \& Cohn, E. (1997). Economies of scale and scope in Japanese private universities. Education Economics, 5(2):107-115.

Issler, J. V. \& Pillar, T. C. L. A. (2002). Mensurando a produção científica de pesquisadores e instituições brasileiras. EPGE/FGV http://www.fgv.br/epge/home/PisDownload/929.pdf [25 Fev. 2003].

Johnes, G. (1997). Costs and industrial structures in contemporary british higher education. The Economic Journal, 107:727-737.

Johnstone, D. B. (1998). The financing and management of higher education: A status report on worldwide reforms. paper financiado pelo World Bank como parte das contribuições para UNESCO - World Conference on Higher Education, 5-9 de outubro.

Koshal, R. K. \& Koshal, M. (1995). Quality and economies of scale in higher education. Applied Economics, 27(8):773-778.

Koshal, R. K. \& Koshal, M. (1999). Economies of scale and scope in higher education: A case of comprehensive universities. Economics of Education Review, $18(2): 269-277$.

Koshal, R. K., Koshal, M., \& Gupta, A. (2001). Multi-product total cost function for higher education: A case of Bible colleges. Economics of Education Review, 20(3):297-303. 
Marinho, A., Resende, M., \& Façanha, L. O. (1997). Brazilian federal universities: Relative efficiency evaluation and data envelopment analysis. Revista Brasileira de Economia, 51(4):489-508.

Psacharopoulos, G. (1996). Public spending on higher education in developing countries: Too much rather than too little. Economics of Education Review, 15(4):421-422.

Verry, D. W. \& Layard, P. R. G. (1975). Cost functions for university teaching and research. The Economic Journal, 85:55-74.

World Bank (1994). Higher education: The lessons of experience. Development in Practice. World Bank, Washington, D.C. 